\title{
Empagliflozin in women with type 2 diabetes and cardiovascular disease - an analysis of EMPA-REG OUTCOME ${ }^{\circledR}$
}

\author{
Bernard Zinman ${ }^{1}$. Silvio E. Inzucchi ${ }^{2} \cdot$ Christoph Wanner $^{3} \cdot$ Uwe Hehnke $^{4} \cdot$ Jyothis T. George $^{4} \cdot$ Odd Erik Johansen $^{5}$. \\ David Fitchett ${ }^{6} \cdot$ on behalf of the EMPA-REG OUTCOME ${ }^{\oplus}$ investigators
}

Received: 28 October 2017 / Accepted: 4 April 2018 / Published online: 30 April 2018

(C) Springer-Verlag GmbH Germany, part of Springer Nature 2018

\begin{abstract}
Aims/hypothesis The global epidemic of type 2 diabetes affects women and men equally; however, the relative impact on the cardiovascular (CV) system appears greater for women than men when compared with peers without diabetes. Furthermore, women are often under-represented in $\mathrm{CV}$ outcome trials, resulting in less certainty about the impact of $\mathrm{CV}$ prevention therapies across the sexes. The EMPA-REG OUTCOME ${ }^{\circledR}$ trial, which included $28.5 \%$ women, found that empagliflozin, given in addition to standard of care, reduced the risk of CV death by $38 \%$, heart failure (HF) hospitalisation by $35 \%$ and a composite endpoint for incident or worsening nephropathy by $39 \%$. Here we report a secondary analysis of the trial to determine the relative effects of empagliflozin in women vs men.

Methods The population studied were individuals with type 2 diabetes $\left(\mathrm{HbA}_{1 \mathrm{c}} 53-86 \mathrm{mmol} / \mathrm{mol}[7-10 \%]\right.$ and eGFR $>30 \mathrm{ml} \mathrm{min}^{-1}[1.73 \mathrm{~m}]^{-2}$ ), with established atherosclerotic CV disease. Individuals were randomised to receive empagliflozin $10 \mathrm{mg}$ or $25 \mathrm{mg}$, or placebo once daily in addition to standard of care, and followed. The trial continued until $\geq 691$ individuals had experienced an adjudicated event included in the primary outcome. All CV outcome events, including HF hospitalisations and deaths were prospectively adjudicated by blinded clinical events committees.

Results At baseline, the demographic profile of the 2004 women (age \pm standard deviation $63.6 \pm 8.8$ years) compared with the 5016 men (age $63.0 \pm 8.6$ years) in the trial was largely similar, with the exception that LDL-cholesterol was numerically higher in women $(2.5 \pm 1.0 \mathrm{vs} 2.1 \pm 0.9 \mathrm{mmol} / \mathrm{l})$, consistent with lower rates of lipid-lowering therapies $(75.4 \%$ vs $83.2 \%)$. Women were also less likely to have smoked (31.5\% vs $69.9 \%)$. The annualised incidence rate for women in the placebo group was numerically lower than in men for $\mathrm{CV}$ death (1.58\% vs $2.19 \%)$, numerically higher for HF hospitalisation (1.75\% vs $1.33 \%)$ and similar for renal events $(7.22 \%$ vs 7.75\%). We did not detect any effect modification by sex within the statistical power restrictions of the analysis for CV death, HF hospitalisation and incident or worsening nephropathy (interaction $p$ values $0.32,0.20$ and 0.85 , respectively). Compared with placebo, empagliflozin increased the rates of genital infections in both women (2.5\% vs $10.0 \%)$ and men (1.5\% vs $2.6 \%)$.

Conclusions/interpretation CV death, HF hospitalisation and incident or worsening nephropathy rate reductions induced by empagliflozin were not different between women and men.
\end{abstract}

Keywords Cardiovascular disease $\cdot$ Heart failure $\cdot$ Mortality $\cdot$ SGLT2 inhibition · Type 2 diabetes · Women

Odd Erik Johansen and David Fitchett are joint senior authors.

Electronic supplementary material The online version of this article (https://doi.org/10.1007/s00125-018-4630-2) contains peer-reviewed but unedited supplementary material, which is available to authorised users.

Odd Erik Johansen

odd_erik.johansen@boehringer-ingelheim.com

1 Lunenfeld-Tanenbaum Research Institute, Mount Sinai Hospital, University of Toronto, Toronto, ON, Canada

2 Section of Endocrinology, Yale University School of Medicine, New Haven, CT, USA
3 Department of Medicine, Division of Nephrology, Würzburg University Clinic, Würzburg, Germany

4 Boehringer Ingelheim Pharma GmbH \& Co. KG, Ingelheim, Germany

5 Boehringer Ingelheim Norway KS, Drengsrudbekken 8, PO Box 405, N-1373 Asker, Norway

6 Division of Cardiology, St Michael's Hospital, University of Toronto, Toronto, ON, Canada 


\section{Research in context}

\section{What is already known about this subject?}

- The global epidemic of type 2 diabetes affects women and men equally; however, the relative cardiovascular impact is greater for women

- The EMPA-REG OUTCOME ${ }^{\circledR}$ trial that included $28.5 \%$ women found that empagliflozin, given in addition to standard care, reduced the risk of cardiovascular (CV) death by $38 \%$, heart failure (HF) hospitalisation by $35 \%$ and a composite endpoint for incident or worsening nephropathy by $39 \%$, while increasing the rates of genital infections two- to threefold

\section{What is the key question?}

- Are there differing effects of empagliflozin between women and men who participated in EMPA-REG OUTCOME ?

\section{What are the new findings?}

- The annualised incidence rate for women vs men in the placebo group was numerically lower for CV death (1.58\% vs $2.19 \%)$, numerically higher for HF hospitalisation (1.75\% vs $1.33 \%$ ) and similar for renal events $(7.22 \%$ vs $7.75 \%)$

- Effects of empagliflozin on reducing clinical outcome events were not different between the sexes

\section{How might this impact on clinical practice in the foreseeable future?}

- In-depth analysis provides reassurance on the use of empagliflozin in both men and women. Consequently, clinicians should consider the reductions in risk of CV death, HF hospitalisation and incident or worsening nephropathy by empagliflozin when choosing therapies for their patients

$\begin{array}{ll}\text { Abbreviations } \\ \text { ACEi } & \text { ACE inhibitor } \\ \text { ARB } & \text { Angiotensin receptor blocker } \\ \text { CV } & \text { Cardiovascular } \\ \text { DBP } & \text { Diastolic blood pressure } \\ \text { HF } & \text { Heart failure } \\ \text { MDRD } & \text { Modification of diet in renal disease } \\ \text { SBP } & \text { Systolic blood pressure }\end{array}$

\section{Introduction}

The global epidemic of type 2 diabetes is expected to affect 642 million individuals by 2040 and impacts women and men equally [1]. It has been postulated that when women develop diabetes, they lose their generally protective effect against cardiovascular (CV) complications. Thus, the relative risk for $\mathrm{CV}$ complications is greater in women with diabetes than men when compared with peers without diabetes. This observation may relate to differences in CV risk factors in men and women, influence of sex hormones on $\mathrm{CV}$ risk, or sex disparities in the management of diabetes and/or CV risk factors [2]. Moreover and disappointingly, women typically only constitute $25-40 \%$ of the study populations in major CV outcome trials [3-5]. Thus, there may be less certainty about efficacy and safety of $\mathrm{CV}$ preventive strategies in women with diabetes. The EMPA-REG OUTCOME ${ }^{\circledR}$ trial, which included $28.5 \%$ women, found that empagliflozin, given in addition to standard of care, reduced the risk of $\mathrm{CV}$ death by $38 \%$ (HR 0.62 [95\% CI $0.49,0.77] ; p<0.001)$, heart failure (HF) hospitalisation by $35 \%$ (HR $0.68[0.57,0.82] ; p<0.001)$ and a composite endpoint for incident or worsening nephropathy by $39 \%$ (HR 0.61 [0.53, 0.70]; $p<0.001)$ vs placebo [5-7]. As a result of this trial, approval for a $\mathrm{CV}$ death prevention indication in individuals with type 2 diabetes and established CV disease was granted by the US Food and Drug Administration and other regulatory authorities. Here we report a secondary prespecified analysis [5] of the trial to determine the relative effects of empagliflozin in women vs men.

\section{Methods}

Description of the trial design (NCT01131676) and methodology used has previously been reported [5-7]. In brief, the population studied was individuals with type 2 diabetes $\left(\mathrm{HbA}_{1 \mathrm{c}}\right.$ 53-86 $\mathrm{mmol} / \mathrm{mol}$ [7-10\%] and eGFR > $30 \mathrm{ml} \mathrm{min}^{-1}[1.73 \mathrm{~m}]^{-2}$ ) who had established atherosclerotic $\mathrm{CV}$ disease. Individuals were randomised to receive empagliflozin $10 \mathrm{mg}$ or $25 \mathrm{mg}$, or placebo once daily in addition to standard of care. Throughout the trial, investigators were encouraged to treat $\mathrm{CV}$ risk factors (including dyslipidaemia and hypertension) to achieve the best available standard of care according to local guidelines. All CV outcome events were 
Table 1 Baseline characteristics by sex in EMPA-REG OUTCOME

\begin{tabular}{|c|c|c|}
\hline & Women & Men \\
\hline Total study population $(N=7020)$ & $2004(28.5)$ & $5016(71.5)$ \\
\hline Age, years & $63.6 \pm 8.8$ & $63.0 \pm 8.6$ \\
\hline BMI, $\mathrm{kg} / \mathrm{m}^{2}$ & $31.2 \pm 5.7$ & $30.4 \pm 5.1$ \\
\hline Weight, kg & $77.9 \pm 17.0$ & $89.7 \pm 18.6$ \\
\hline Waist circumference, $\mathrm{cm}$ & $102.1 \pm 14.0$ & $105.9 \pm 13.5$ \\
\hline \multicolumn{3}{|l|}{ Type 2 diabetes characteristics } \\
\hline $\mathrm{HbA}_{1 \mathrm{c}}, \mathrm{mmol} / \mathrm{mol}$ & $65.5 \pm 9.7$ & $64.4 \pm 9.0$ \\
\hline $\mathrm{HbA}_{1 \mathrm{c}}, \%$ & $8.1 \pm 0.9$ & $8.0 \pm 0.8$ \\
\hline$>10$ years since diabetes diagnosis, $\%$ & 57.8 & 56.9 \\
\hline \multicolumn{3}{|l|}{ Glucose-lowering agents, $\%$} \\
\hline No treatment & 1.8 & 1.8 \\
\hline 1 treatment & 31.1 & 28.8 \\
\hline 2 treatments & 50.3 & 47.8 \\
\hline$\geq 3$ treatments & 14.6 & 18.4 \\
\hline Any metformin & 73.5 & 74.2 \\
\hline Any sulfonylurea & 41.0 & 43.5 \\
\hline Any insulin & 51.0 & 47.1 \\
\hline Any glitazone & 2.8 & 4.8 \\
\hline Any DPP4 inhibitor & 8.2 & 12.6 \\
\hline Any GLP-1 receptor agonist & 2.4 & 2.9 \\
\hline \multicolumn{3}{|l|}{ CV history and risk factors, $\%$} \\
\hline \multicolumn{3}{|l|}{ Smoking } \\
\hline Never & 68.5 & 30.1 \\
\hline Current & 9.2 & 14.9 \\
\hline Coronary artery disease ${ }^{a}$ & 62.8 & 80.7 \\
\hline History of myocardial infarction & 36.7 & 50.6 \\
\hline Coronary artery bypass grafting & 16.7 & 28.0 \\
\hline History of stroke & 31.6 & 20.0 \\
\hline Peripheral arterial disease & 22.6 & 20.1 \\
\hline Heart failure & 10.5 & 9.9 \\
\hline $\mathrm{eGFR}^{\mathrm{b}}, \mathrm{ml} \mathrm{min}^{-1}[1.73 \mathrm{~m}]^{-2}$ & $72.7 \pm 22.2$ & $74.6 \pm 21.1$ \\
\hline 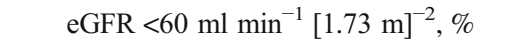 & 29.2 & 24.6 \\
\hline Systolic BP, mmHg & $136.0 \pm 17.6$ & $135.2 \pm 16.8$ \\
\hline Diastolic BP, mmHg & $76.0 \pm 10.0$ & $76.9 \pm 9.8$ \\
\hline LDL-cholesterol, mmol/1 & $2.5 \pm 1.0$ & $2.1 \pm 0.9$ \\
\hline \multicolumn{3}{|l|}{ Albuminuria, $\%$} \\
\hline Normoalbuminuria & 64.0 & 57.6 \\
\hline Microalbuminuria & 26.1 & 29.7 \\
\hline Macroalbuminuria & 9.2 & 11.7 \\
\hline Missing & 0.7 & 1.0 \\
\hline \multicolumn{3}{|l|}{ CV pharmacotherapy, $\%$} \\
\hline Any BP lowering agent & 94.3 & 95.2 \\
\hline 0 BP-lowering agent & 5.7 & 4.8 \\
\hline 1 BP-lowering agent & 19.0 & 18.2 \\
\hline 2 BP-lowering agents & 29.9 & 32.4 \\
\hline 3 BP-lowering agents & 27.9 & 25.5 \\
\hline$\geq 4$ BP-lowering agents & 17.5 & 19.1 \\
\hline ACEi/ARB & 79.7 & 81.1 \\
\hline Diuretic agents & 47.6 & 41.5 \\
\hline
\end{tabular}

Table 1 (continued)

\begin{tabular}{lll}
\hline & Women & Men \\
\hline Beta blockers & 58.9 & 67.3 \\
Digitalis & 2.1 & 3.1 \\
Lipid-lowering therapies & 75.4 & 83.2 \\
Any statins & 71.1 & 79.3 \\
Any fibrate & 7.7 & 9.5 \\
Platelet inhibitors & 80.9 & 87.7 \\
Any ASA & 77.5 & 84.7 \\
Any clopidogrel & 10.6 & 10.6 \\
\hline
\end{tabular}

Data are given as $n(\%)$ or mean $\pm \mathrm{SD}$, as shown

${ }^{\text {a }}$ Coronary artery disease defined as any of the components of history of myocardial infarction, coronary artery bypass graft, multivessel coronary artery disease, single vessel coronary artery disease

${ }^{\mathrm{b}}$ eGFR estimated using MDRD formula

ASA, acetylsalicylic acid; DPP4, dipeptidyl peptidase-4; GLP-1, glucagon-like peptide 1

prospectively adjudicated by independent and blinded clinical events committees. For the renal outcomes analysed, serum creatinine and urinary albumin in spot urine samples obtained during regular study visits were measured in central laboratories with the use of standardised procedures. We used the modification of diet in renal disease (MDRD) formula to estimate the eGFR. Safety was assessed on the basis of adverse events that occurred during treatment or within 7 days after the last dose of study drug, and collected as previously reported [5-7].

Cox proportional hazards models were used to assess between-group differences in the risk of an outcome after adjustment for study group, age, sex, baseline BMI, $\mathrm{HbA}_{1 \mathrm{c}}$, eGFR and region. We present overall $\mathrm{HR}$ according to sex and the interaction $p$ value considering the interaction of treatment $x$ sex for CV death, HF hospitalisation and the predefined composite renal outcome. Kaplan-Meier plots are presented to display events over time; adverse events are expressed as proportions. Post hoc power considerations were derived using ADDPLAN (version 6.1.1, 2014, ICON, www. iconplc.com/innovation/addplan). All study participants gave informed consent prior to enrolment, and the investigations were approved by ethics committees or institutional review boards.

\section{Results}

The profile at baseline (Table 1, electronic supplementary material [ESM] Table 1) was largely similar between the 2004 women in the trial (age [mean $\pm \mathrm{SD}$ ], $63.6 \pm 8.8$ years; 
a

3-point MACE
All patients
Sex
Women
Men
CV death
All patients
Sex
Women
Men
Hospitalisation for heart failure
All patients
Sex
Women
Men
Incident or worsening nephropathy
All patients
Sex
Women
Men

b

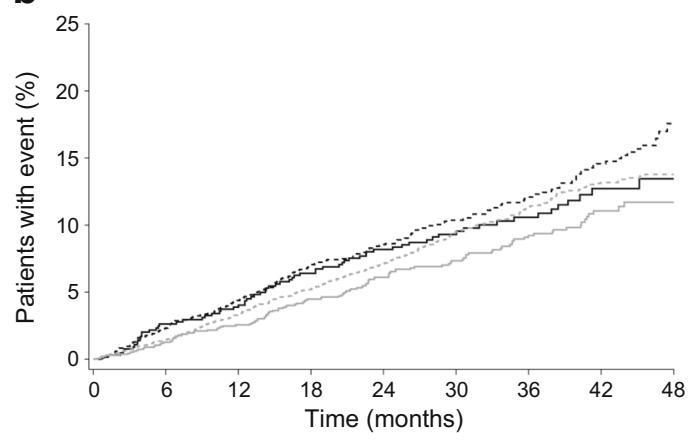

Patients at risk Placebo, women Placebo, men $\begin{array}{lllllllll}1680 & 1629 & 1581 & 1524 & 1343 & 994 & 848 & 552 & 128\end{array}$

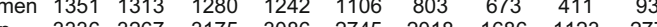

d

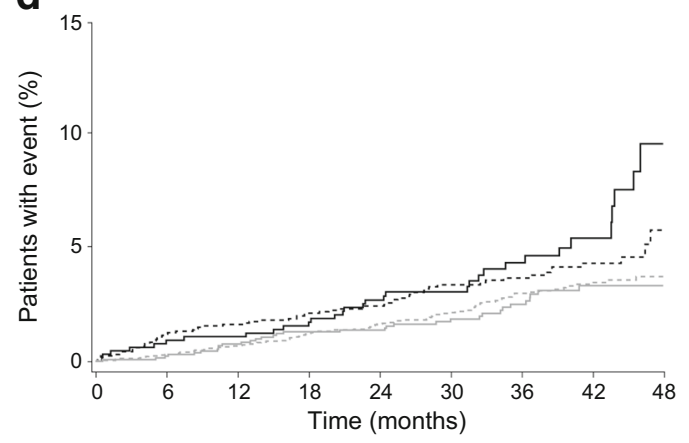

Patients at risk

$\begin{array}{llllllllll}\text { Placebo, women } & 653 & 637 & 624 & 608 & 548 & 403 & 326 & 202 & 39\end{array}$ $\begin{array}{llllllllll}\text { Placebo, men } & 1680 & 1634 & 1602 & 1565 & 1384 & 1021 & 876 & 573 & 129\end{array}$ $\begin{array}{llllllllll}\text { Empagliflozin, women } & 1351 & 1322 & 1294 & 1263 & 1140 & 830 & 699 & 429 & 93\end{array}$ $\begin{array}{llllllllll}\text { Empagliflozin, men } & 3336 & 3292 & 3229 & 3164 & 2848 & 2120 & 1788 & 1205 & 302\end{array}$

BMI $31.2 \pm 5.7 \mathrm{~kg} / \mathrm{m}^{2}$; systolic/diastolic blood pressure [SBP/DBP] $136.0 \pm 17.6 / 76.0 \pm 10.0 \mathrm{mmHg}$; eGFR $72.7 \pm$ $22.2 \mathrm{ml} \mathrm{min}^{-1}[1.73 \mathrm{~m}]^{-2} ; 64 \%$ normoalbuminuria), and the 5016 men (age $63.0 \pm 8.6$; BMI $30.4 \pm 5.1$; SBP/DBP 135.2
Placebo

$\operatorname{HR}(95 \% \mathrm{Cl})$

Treatment

by sex

interaction

$p=0.8114$

$p=0.3219$

$30 / 653$ (4.6)

$107 / 1680(6.4)$

95/2333 (4.1)

$32 / 653$ (4.9)

$63 / 1680$ (3.8)

$388 / 2061$ (18.8)

$105 / 596(17.6)$

283/1465 (19.3)
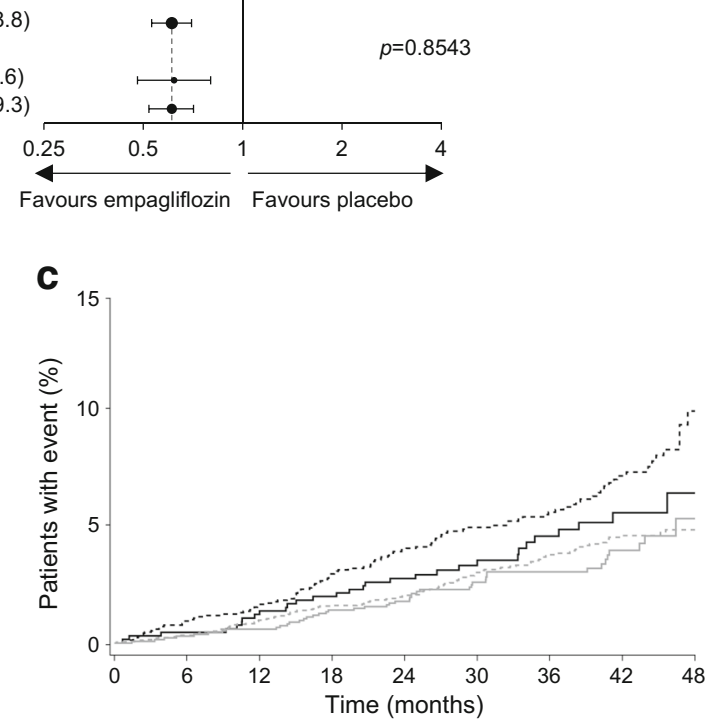

Patients at risk

$\begin{array}{llllllllll}\text { Placebo, women } & 653 & 646 & 637 & 628 & 574 & 427 & 352 & 215 & 40\end{array}$ $\begin{array}{llllllllll}\text { Placebo, men } & 1680 & 1657 & 1643 & 1615 & 1438 & 1076 & 929 & 610 & 137\end{array}$ $\begin{array}{lllllllllll}\text { Empagliflozin, women } & 1351 & 1336 & 1324 & 1308 & 1182 & 876 & 746 & 464 & 104\end{array}$ $\begin{array}{llllllllll}\text { Empagliflozin, men } & 3336 & 3315 & 3284 & 3248 & 2946 & 2203 & 1871 & 1258 & 310\end{array}$

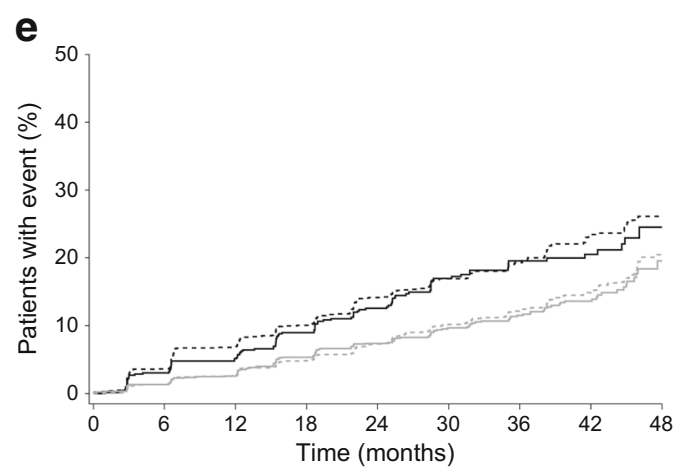

Patients at risk

$\begin{array}{lccccccccc}\text { Placebo, women } & 596 & 560 & 530 & 495 & 419 & 283 & 223 & 134 & 27\end{array}$ $\begin{array}{llllllllll} & 1465 & 1386 & 1306 & 1208 & 1014 & 733 & 610 & 387 & 79\end{array}$ $\begin{array}{llllllllll}\text { Empagliflozin, women } & 1200 & 1146 & 1098 & 1037 & 886 & 630 & 524 & 313 & 68 \\ \text { Empagliflozin, men } & 2924 & 2848 & 2750 & 2632 & 2285 & 1649 & 1363 & 906 & 222\end{array}$

$\pm 16.8 / 76.9 \pm 9.8 ;$ eGFR $74.6 \pm 21.1 ; 57.6 \%$ normoalbuminuria). The geographic representation in the trial was also similar between the sexes (women: $41.0 \%$ Europe; $17.8 \%$ North America; $18.9 \%$ Latin 
Fig. 1 (a) Forest plot of 3-point Major Adverse Cardiovascular Events (MACE [composite of CV death, nonfatal myocardial infarction or nonfatal stroke]), CV death, HF hospitalisation and nephropathy by sex, and interaction $p$ values. Values were determined by Cox regression analysis in patients treated with $\geq 1$ dose of study drug. (b-e) KaplanMeier estimates in patients treated with $\geq 1$ dose of study drug. Solid grey line, women in the empagliflozin group; dashed grey line, men in the empagliflozin group; solid black line, women in the placebo group; dashed black line, men in the placebo group. (b) 3-point MACE over time in women and men with empagliflozin vs placebo. HR (95\% CI): women, $0.83(0.62,1.11)$; men, $0.87(0.73,1.02) . p=0.8114$ for treatment by sex interaction. (c) $\mathrm{CV}$ death over time in women and men with empagliflozin vs placebo. HR (95\% CI): women, $0.76(0.48,1.20)$; men, $0.58(0.45,0.75) . p=0.3219$ for treatment by sex interaction. (d) HF hospitalisation over time in women and men with empagliflozin vs placebo. HR (95\% CI): women, 0.50 (0.31, 0.81); men, $0.73(0.53$, 1.01). $p=0.1981$ for treatment by sex interaction. (e) Occurrence of worsening of nephropathy (progression to macroalbuminuria, doubling of serum creatinine, initiation of renal-replacement therapy, or death from renal disease) over time in women and men with empagliflozin vs placebo). HR (95\% CI): women, 0.62 (0.48, 0.80); men, 0.61 (0.52, $0.71) . p=0.8543$ for treatment by sex interaction

America; $18.1 \%$ Asia; $4.2 \%$ South Africa vs men: $41.1 \%$ Europe; $20.7 \%$ North America; $14.0 \%$ Latin America; $19.6 \%$ Asia; $4.5 \%$ South Africa). In addition, type 2 diabetes disease characteristics were also largely similar: $\mathrm{HbA}_{1 \mathrm{c}} 65.5 \pm 9.7 \mathrm{mmol} / \mathrm{mol}(8.1 \% \pm 0.9 \%)$ vs $64.4 \pm$ $9.0 \mathrm{mmol} / \mathrm{mol}(8.0 \% \pm 0.8 \%)$; type 2 diabetes duration > 10 years: $57.8 \%$ vs $56.9 \%$; and any use of metformin ( $73.5 \%$ vs $74.2 \%$ ), sulfonylurea ( $41.0 \%$ vs $43.5 \%$ ) or insulin $(51.0 \%$ vs $47.1 \%)$.

LDL-cholesterol was, however, numerically higher in women $(2.5 \pm 1.0$ vs $2.1 \pm 0.9 \mathrm{mmol} / \mathrm{l})$, likely reflecting lower rates of lipid-lowering therapies ( $75.4 \%$ vs $83.2 \%$ ), in particular statin use $(71.1 \%$ vs $79.3 \%)$. Women also had lower prevalent use of anti-platelet therapies $(80.9 \%$ vs $87.7 \%)$ and were less likely to have smoked $(31.5 \%$ vs $69.9 \%$ ). Use of antihypertensive drugs were similar ( $75.3 \%$ vs $77.0 \%$ used $\geq$ two antihypertensive agents), including use of angiotensin converting enzyme inhibitors (ACEis) / angiotensin receptor blockers (ARBs) $(79.7 \%$ vs $81.1 \%)$, but slightly more women used diuretics $(47.6 \%$ vs $41.5 \%)$ and slightly fewer women used beta blockers (58.9\% vs $67.3 \%$ ). Throughout the trial (ESM Table 2), a higher percentage of patients in the placebo group, for both men and women, received additional antihypertensive therapies (women: $49.8 \%$ [placebo] vs $42.9 \%$ [empagliflozin]; men: $51.5 \%$ vs $45.2 \%$ ) and acetylsalicylic acid, with the latter occurring numerically more frequent in women (women: $21.7 \%$ vs $17.7 \%$; men: $18.6 \%$ vs $18.0 \%$ ), whereas new introduction of statin therapy was similar (women: $25.7 \%$ vs $24.9 \%$; men: $25.8 \%$ vs $24.2 \%$ ).

Prior coronary artery disease was less common in women ( $62.8 \%$ vs $80.7 \%$ ), but women had a more frequent history of stroke (31.6\% vs $20.0 \%)$. History of HF was similar (10.5\% vs $9.9 \%)$.
Effects of empagliflozin on reducing $\mathrm{BP}, \mathrm{HbA}_{1 \mathrm{c}}$, weight and waist circumference were of similar magnitude regardless of sex (data not shown). The annualised incidence rate for women in the placebo group was numerically lower than that in men for CV death (1.58\% vs $2.19 \%)$, numerically higher for HF hospitalisation ( $1.75 \%$ vs $1.33 \%$ ), and similar for renal events (7.22\% vs $7.75 \%)$. Empagliflozin reduced the primary composite endpoint (CV death, non-fatal stroke, non-fatal myocardial infarction) relatively by $14 \%$ (Fig. 1a,b) by a similar degree, irrespective of sex (interaction $p$ value 0.8114 ). We did not detect any effect modification by sex within the statistical power restrictions of the analysis for $\mathrm{CV}$ death, HF hospitalisation and incident or worsening nephropathy (Fig. 1a, c-e; interaction $p$ values $0.32,0.20$ and 0.85 , respectively). Separation of the incidence curves for these events were generally early and risk reduction with empagliflozin vs placebo persisted for the trial's duration (Fig. 1b-e). Post hoc power considerations are provided in ESM Table 3.

Empagliflozin was well tolerated by women, with no remarkable difference between empagliflozin and placebo for most adverse events assessed, including urinary tract infections. Genital infections, which were more common in women than in men in both treatment groups, were increased with empagliflozin treatment. In women this was observed in $2.5 \%$ in the placebo group and $10.0 \%$ in the empagliflozin group while in men, $1.5 \%$ and $2.6 \%$, respectively, consistent with the known safety profile of sodium-glucose cotransporter-2 (SGLT2) inhibition. Nevertheless, discontinuation of the study drug due to this adverse event was infrequent. For every 1000 women treated with empagliflozin for 3.1 years, there occurred 12 fewer CV deaths, 27 fewer hospitalised HFs and 83 fewer incident or worsening nephropathy events, with 101 more genital infections, in comparison with placebo.

\section{Discussion}

Women with type 2 diabetes and established CV disease who were enrolled in the EMPA-REG OUTCOME ${ }^{\circledR}$ trial experienced high $\mathrm{CV}$ and renal event rates, in particular hospitalisation for HF that occurred numerically more frequently than in men, emphasising the high impact of diabetes on complications in this group. The numerically higher event rate for HF hospitalisation in women than in men is interesting in light of a similar between-sexes a priori 5 year estimated HF risk using the Health ABC HF risk score [8], bearing in mind the limitation that our trial was not powered to address sex differences. This observation could be related both to underlying factors specific to women [2] or, as suggested by others [9], because evidence-based HF therapies are used less often by women. In EMPA-REG 
OUTCOME ${ }^{\circledR}$, use of glucose-lowering medications, ACEis/ARBs and overall antihypertensive therapy use (albeit characterised by somewhat more diuretic and somewhat less beta-blocker use), was similar in men and women. However, both women and men allocated to placebo had a higher proportion of new prescriptions of such drugs during the trial than those allocated to empagliflozin. Women did have lower use of lipid-lowering and anti-platelet therapies, but neither of these have been associated with HF outcomes. Also, as fewer women than men were current or former smokers, a habit usually associated with increased HF risk [10], our observation could lend support to the hypothesis of female-specific factors playing a role. Empagliflozin was generally well tolerated, with a higher frequency of genital infections in both sexes, more so both in relative and absolute terms in women. This is congruent with results from previous reports on the use of empagliflozin, which has increased the risk of yeast vaginitis in earlier trials involving women with type 2 diabetes at lower CV risk [11].

In conclusion, $\mathrm{CV}$ death, HF hospitalisation and incident or worsening nephropathy rate reductions induced by empagliflozin were not different between women and men.

Acknowledgements The authors thank the individuals who participated in this trial and I. Wiener and A. Elsaesser (Biostatistics and Data Sciences, Boehringer Ingelheim, Germany) for ADDPLAN post hoc power calculations.

Data availability The datasets generated during and/or analysed during the current study are available from the corresponding author on reasonable request.

Funding The EMPA-REG OUTCOME ${ }^{\circledR}$ trial was funded by the Boehringer Ingelheim \& Eli Lilly and Company Diabetes Alliance.

Duality of interest BZ has received research grants awarded to his institution from Boehringer Ingelheim, AstraZeneca and NovoNordisk; honoraria from Janssen, Sanofi and Eli Lilly and Company; and honoraria from Boehringer Ingelheim, NovoNordisk and Merck. SEI reports honoraria from AstraZeneca, Boehringer Ingelheim, Daiichi-Sankyo, Eisai, Janssen, Intarcia Therapeutics, Inc., Novo Nordisk, Sanofi/Lexicon, vTv Therapeutics. CW reports honoraria from Bayer, Boehringer Ingelheim, Janssen and Sanofi. UH, JTG and OEJ are employed by Boehringer Ingelheim. DF reports honoraria from Sanofi, Merck \& Co., Amgen, AstraZeneca, Eli Lilly and Company and Boehringer Ingelheim.
Contribution statement All authors fulfil the International Committee of Medical Journal Editors (ICMJE) criteria for authorship, are fully responsible for all content and editorial decisions, were involved at all stages of manuscript development and have approved the final version. Representatives of Boehringer Ingelheim (UH, JTG, OEJ) were involved in the study design, collection, analysis and interpretation of data; writing the report and the decision to submit the report for publication. BZ is the guarantor of this work.

\section{References}

1. International Diabetes Federation (2015) IDF Diabetes Atlas (7th edition). Available from https://www.idf.org/e-library/ epidemiology-research/diabetes-atlas/13-diabetes-atlas-seventhedition.html. Accessed 20 Oct 2017

2. Lyon A, Jackson EA, Kalyani RR, Vaidya D, Kim C (2015) Sexspecific differential in risk of diabetes-related macrovascular outcomes. Curr Diab Rep 15:85

3. Kim ES, Menon V (2009) Status of women in cardiovascular clinical trials. Arterioscler Thromb Vasc Biol 29:279-283

4. Marx N, Rosenstock J, Kahn SE et al (2015) Design and baseline characteristics of the CARdiovascular outcome trial of LINAgliptin versus glimepiride in type 2 diabetes: CAROLINA ${ }^{\circledR}$. Diab Vasc Dis Res 12:164-174

5. Zinman B, Wanner C, Lachin JM et al (2015) Empagliflozin, cardiovascular outcomes, and mortality in type 2 diabetes. $\mathrm{N}$ Engl $\mathrm{J}$ Med 373:2117-2128

6. Fitchett D, Zinman B, Wanner C et al (2016) Heart failure outcomes with empagliflozin in patients with type 2 diabetes at high cardiovascular risk: results of the EMPA-REG OUTCOME ${ }^{\circledR}$ trial. Eur Heart J 37:1526-1534

7. Wanner C, Inzucchi SE, Lachin JM et al (2016) Empagliflozin and progression of kidney disease in type 2 diabetes. N Engl J Med 375: 323-334

8. Fitchett D, Butler J, van de Borne P et al (2018) Effects of empagliflozin on risk for cardiovascular death and heart failure hospitalisation across the spectrum of heart failure risk in the EMPA-REG OUTCOME ${ }^{\circledR}$ Trial. Eur Heart J 39:363-370

9. Johansson I, Dahlström U, Edner M, Näsman P, Rydén L, Norhammar A (2015) Risk factors, treatment and prognosis in men and women with heart failure with and without diabetes. Heart 101:1139-1148

10. Gopal DM, Kalogeropoulos AP, Georgiopoulou VV et al (2012) Cigarette smoking exposure and heart failure risk in older adults: the health, aging, and body composition study. Am Heart J 164: 236-242

11. Devi R, Mali G, Chakraborty I, Unnikrishnan MK, Abdulsalim S (2017) Efficacy and safety of empagliflozin in type 2 diabetes mellitus: a meta-analysis of randomized controlled trials. Postgrad Med 129:382-392 